\title{
Chronische Rhinosinusitis - ein häufiges Puzzleteil in der Pneumologie
}

\author{
Chronic Rhinosinusitis - \\ A Common Piece in Pneumological Puzzles
}

H. Knoop ${ }^{1,2}$, U. Knoop 2 , S. Dazert ${ }^{3}$, S. Ewig' ${ }^{1}$, C. M. Heyer ${ }^{4}$, A. Tannapfel ${ }^{5}$, A. Gurr ${ }^{3}$

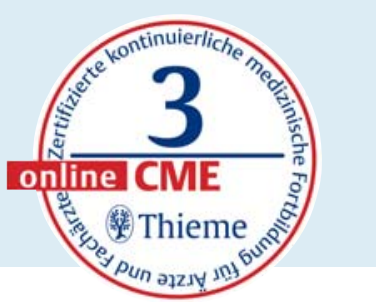

Die Institute sind am Ende des Artikels gelistet.

VNR

2760512013141214313

Bibliografie

Dol http://dx.doi.org/

10.1055/s-0032-1326096

Pneumologie 2013; 67: 162-173

(c) Georg Thieme Verlag KG

Stuttgart · New York

ISSN 0934-8387

Korrespondenzadresse

Dr. med. Heiko Knoop

Medizinische Klinik III -

Pneumologie, Allergologie,

Schlaf- und Beatmungsmedizin

Berufsgenossenschaftliches

Universitätsklinikum

Bergmannsheil GmbH

Universitätsklinikum der

Ruhr-Universität Bochum

Bürkle-de-la-Camp-Platz 1

44789 Bochum

Heiko.Knoop@ruhr-uni-

bochum.de

\section{Lernziel}

Die chronische Rhinosinusitis (CRS) ist eine häufige Erkrankung mit hoher Korrelation zu pneumologischen Krankheitsbildern. Aus diesem Grund ist es für den Pneumologen essenziell, dieses Krankheitsbild zu kennen. In dieser Arbeit werden daher die aktuellen diagnostischen und therapeutischen Optionen unter Berücksichtigung der aktuellen Leitlinien vorgestellt.

\section{Einleitung}

Die chronische Entzündung der Nasenschleimhaut und Mukosa der Nasennebenhöhlen (NNH) wird unter dem Begriff chronische Rhinosinusitis (CRS) zusammengefasst. Während die Rolle der Nasenschleimhaut als Filter der Atemluft einleuchtet, ist die Bedeutung der NNH, deren Entwicklung erst am Ende der Kleinkindphase einsetzt, bis heute nicht gänzlich geklärt. Es werden vor allem Funktionen als Resonanzkörper für die Artikulation und Stimmbildung diskutiert. Obwohl belastbare Daten zur Epidemiologie in Europa fehlen, kann festgehalten werden, dass es sich bei der CRS um eine häufige Erkrankung handelt.

\section{CRS in der Pneumologie}

Chronische Rhinitis und Sinusitis treten für gewöhnlich gemeinsam auf. Die korrekte Bezeichnung ist daher CRS [1]. Die CRS ist ein häufiges Krankheitsbild mit hoher sozioökonomischer Bedeutung [2], dem man auch in der Pneumologie oft begegnet. Die CRS kann Ursache von Symptomen sein, die in unserem Fachgebiet weiter abgeklärt werden sollen (Beispiel chronischer Husten), Teil eines Symptomenkomplexes sein (Beispiel Churg-Strauss-Syndrom) oder auch mit anderen pneumologischen Krankheitsbildern vergesellschaftet sein (Beispiele Asthma bronchiale, zystische Fibrose).

\section{CRS und chronischer Husten}

„Postnasal drip“ im Rahmen einer CRS ist eine der drei häufigsten Ursachen für chronischen Husten und sollte entsprechend im Rahmen der differenzialdiagnostischen Abklärung berücksichtigt werden [3]. Wichtig ist der Zusammenhang mit gastroösophagealem Reflux, der sich nach extraösophageal ausbreitet - sogenannter laryngopharyngealer Reflux - [4], eo ipso eine weitere häufige Ursache für chronischen Husten darstellt und darüber hinaus eine CRS unterhalten kann [5].

\section{CRS und Churg-Strauss-Syndrom (CSS)}

Die CRS zählt zu den Klassifikationskriterien des CSS [6,7], die aktuell überarbeitet werden [8]. Ein Zusammenhang zwischen der Einnahme des Leukotrienantagonisten Montelukast, der in der Therapie des Asthma bronchiale sowie der CRS Verwendung findet, und CSS wird angenommen [9].

\section{CRS und Asthma bronchiale}

Der Zusammenhang zwischen Aspirin ${ }^{\circledR}$-sensitivem Asthma und CRS ist gut belegt $[10,11]$. In diesen Kontext passt auch, dass die häufig mit einem Asthma bronchiale einhergehende Vocal Cord-Dysfunction (VCD) durch einen postnasalen Drip ausgelöst werden kann [12]. Oft wird dieses Zusammenspiel zwischen CRS und Affektion der unteren Atemwege unter dem Begriff sinubronchiales Syndrom zusammengefasst, für den allerdings keine einheitliche Definition existiert. Der Zusammenhang zwischen CRS und der chronisch-obstruktiven Lungenerkrankung (COPD) wurde bislang nicht systematisch untersucht [1].

\section{CRS und zystische Fibrose}

CRS tritt häufig bei Patienten mit zystischer Fibrose (CF) auf [13]. Durch Verschleppung von Keimen aus den NNH kann es insbesondere auch bei lungentransplantierten CF-Patienten zur mikrobiellen Kolonisation der unteren Atemwege kommen [14]. Eine Korrelation zwischen CRS und obstruktivem Schlafapnoesyndrom wurde darüber hinaus bei Kindern und Jugendlichen mit CF be- 
schrieben [15]. Interessanterweise zählen Rhinosinusitiden auch zu den häufigsten unerwünschten Wirkungen unter dem neuen Medikament Ivacaftor, das seit Mitte 2012 für die Behandlung bestimmter CF-Patienten zugelassen ist [16]. In jedem Fall sollte also ein HNO-Arzt zum Team gehören, das CF-Patienten betreut.

\section{CRS und Sarkoidose}

CRS stellt eine häufige Form der extrapulmonalen Sarkoidose dar [17]. Im Rahmen der Umfelddiagnostik sollte daher bei Patienten mit vermuteter oder bestätigter Sarkoidose an die Möglichkeit eines NNH-Befalls gedacht werden.

Neben den typischen CRS-Symptomen wurde vor allem auch nasale Krustenbildung als Zeichen der Schleimhautatrophie beobachtet [18].

Eine sinunasale Schleimhautbiopsie sollte zur Diagnosesicherung erwogen werden [17].

\section{Beatmungs-assoziierte Sinusitis}

Eine VAS (ventilator-associated sinusitis) ist eine häufige Komplikation bei invasiv und endonasal über einen endotrachealen Tubus beatmeten schwer kranken Patienten [19]. Ein Zusammenhang mit der Entstehung nosokomial-erworbener Pneumonien wird diskutiert [20,21]. Eine Korrelation zwischen Beatmung via Tracheostoma und CRS wurde allerdings nicht beschrieben. Zählt man die nCPAP (nasal continuous positive airway pressure)-Therapie zu den nicht-invasiven Beatmungsformen, so wurde ein entsprechender $\mathrm{Zu}$ sammenhang mit der Sinusitis beobachtet [22].

\section{Definition und Einteilung}

Die CRS ist nach aktuellem europäischem Positionspapier folgendermaßen (siehe $\bullet$ Tab. 1) definiert [1].

Die o.g. Beschwerden müssen im Anamnesegespräch explizit abgefragt werden. Eine allergologische Anamneseerhebung nach Symptomen wie Niesen, Fließschnupfen, Nasen-/Augenjucken, Augenbrennen ist ferner obligater Bestandteil der Befragung.

Die idiopathische CRS wird in eine Form mit (Polyposis nasi et sinuum) und eine ohne Nasenpolypen unterteilt (CRS with nasal polyps, CRSwNP; CRS sine nasal polyps, CRSsNP) [1,23]. Die CRSwNP ist durch eine eosinophile Entzündungsreaktion und eine $\mathrm{TH} 2$ (T-Helfer)-Zellantwort charakterisiert. Vor allem bei der CRSwNP gibt es einen Hinweis auf einen Zusammenhang mit Staphylococcus aureus (s. nächster Abschnitt). Bei der CRSsNP dominiert eine neutrophile Entzündungsreaktion und eine TH1-Zellantwort. Im Vergleich zur CRSwNP sind die pathophysiologischen Zusammenhänge bei der CRSsNP weniger untersucht.

Zur Diagnosestellung einer CRS werden zunächst klinische Kriterien verwendet.

\section{Prädisponierende Faktoren}

Grundsätzlich wird angenommen, dass der enge und verwinkelte Aufbau des gesamten Nasennebenhöhlensystems die Drainage und Belüftung der NNH einschränken kann und somit Entzündungsprozesse begünstigt [24]. Evidenz für eine Korrelation zwischen anatomischen Varianten im Bereich der NNH im Allgemeinen und der Inzidenz der CRS gibt es allerdings nicht [1]. Ein Zusammenhang zwischen Allergien und CRS ist nicht bewiesen [25]. Die Beziehung von Asthma bronchiale und CRSwNP gilt hingegen ebenso als bestätigt wie jene zwischen Analgetikaintoleranz und CRSwNP [1]. Eine Studie an Rhinitikern zeigte, dass fehlendes Nasenhaar das Asthmarisiko erhöhte [26]. Gegenstand von Untersuchungen sind vor allem Biofilme [27] - insbesondere durch Staphylococcus aureus -, die ebenso wie die intrazelluläre bakterielle Besiedelung die Resistenz gegenüber den Standardtherapien erhöht $[1,28]$. Staphylokokkenenterotoxine fungieren als Superantigene, die die eosinophile Entzündung verstärken und eine polyklonale IgE-Synthese induzieren (disease modifier). Unklar ist, ob die Biofilme Ursache oder Folge der Schleimhautveränderung bei CRS sind $[29,30]$.

Genetische Einflüsse sind ebenso wie die Bedeutung einer Passivrauchexposition in der Kindheit und Jugend nicht abschließend untersucht [31].

\section{Mikrobielle Erreger}

Die Rolle von Bakterien beim initialen Entstehen einer CRS bleibt unklar, obwohl seit langem spekuliert wird, dass eine nicht ausreichend behandelte bakterielle akute Rhinosinusitis zur Entstehung einer CRS führt [1]. Fest steht, dass Bakterien eine akute Exazerbation einer CRS auslösen können. Bei der CRS kommen vor allem Staphylococcus epidermidis und aureus sowie nichthämolysierende Streptokokken, Moraxella catarrhalis und pathogene Haemophilus species, Anaerobier und Pseudomonas aeruginosa vor [23].

Pilze spielen bei Immuninkompetenten eine Rolle und können eine akute invasive Pilz-CRS (acute invasive fungal rhinosinusits, AIFRS) auslösen [1]. Immuninkompetent können Immunsupprimierte vor allem nach Organtransplantationen, aber auch Patienten mit einem unzureichend kontrollierten Diabetes mellitus sein. Hinsichtlich des Erregerspektrums muss vor allem an Aspergillusund Mucor-Arten gedacht werden.

\section{Diagnostik}

$\nabla$

Die Diagnosestellung beruht in erster Linie auf der Anamneseerhebung mit den typischen Symptomen. Zusätzlich zu den in Tab. 1 genannten Beschwerden können Symptome wie Halsschmerzen, Husten, allgemeines Krankheitsgefühl und Dysphonie vorkommen [23]. Da die rein klinische Diagnostik nicht ausreichend valide ist,

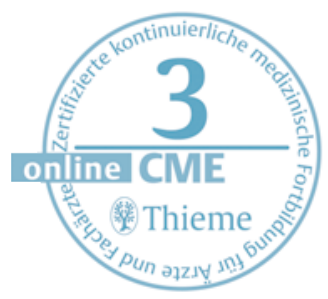


Tab. 1 Klinische Definition der CRS beim Erwachsenen nach EPOS (European Position Paper on Rhinosinusitis and Nasal Polyps) [1].

Klinische Definition der CRS nach EPOS
CRS mit oder ohne Nasenpolypen mit mindestens zwei der nachfolgenden Symptomen,
von denen wiederum eines 1 . oder 2. sein muss:
1. nasale Obstruktion/Schwellung
2. Nasensekretion (anterior/posterior nasal drip)
3. Gesichtsschmerz/Druckgefühl
4. Hyp-oder Anosmie über einen Zeitraum $\geq 12$ Wochen

Abb. 1 Computertomografisch unauffälliger Befund der symmetrisch belüfteten Nasennebenhöhlen, keine relevanten Schleimhautveränderungen, keine ossären Läsionen.

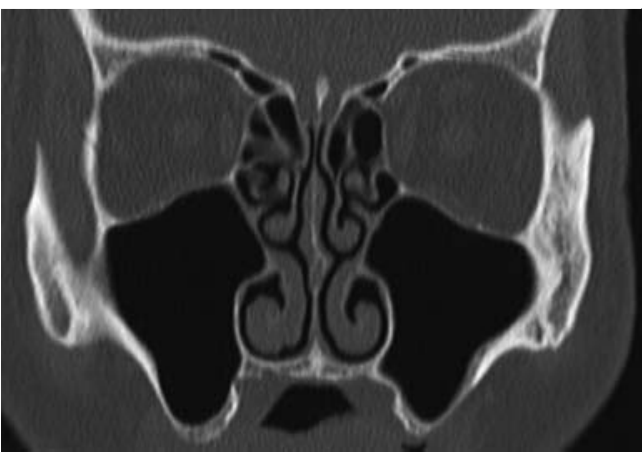

Abb.2 Computertomografischer Nachweis einer kissenförmigen Schleimhautschwellung am Boden des rechten Sinus maxillaris (Pfeil) bei ansonsten unauffälliger Belüftung der Nasennebenhöhlen.

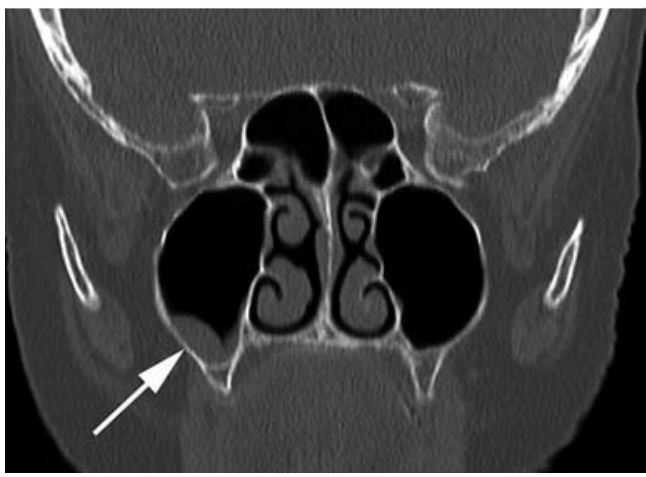

Abb.3 Ausgedehnte Schleimhautschwellungen in beiden Sinus maxillares, in einzelnen Ethmoidalzellen und an den Conchae nasales als Ausdruck einer Sinusitis.

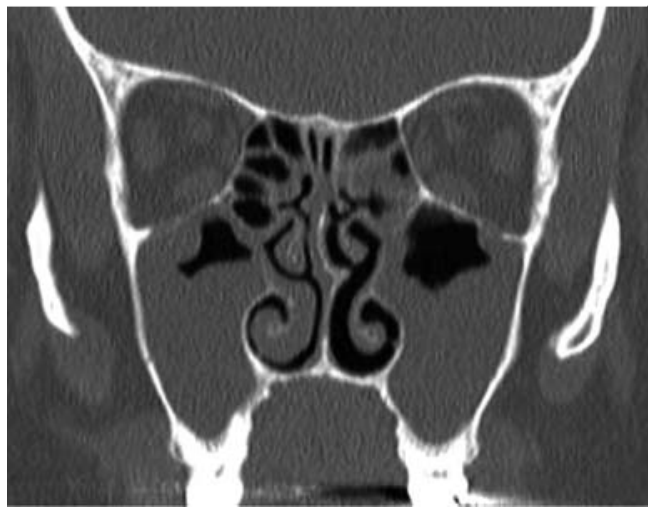

sind für eine sichere Diagnose computertomografische und endoskopische Befunde erforderlich [23]. Die Nasenendoskopie ist hierbei nicht nur für die Diagnostik - z.B. Abklärung von Nasenpolypen, gezielte Gewinnung von Material zur mikrobiologischen Diagnostik - hilfreich, sondern auch für die Therapieplanung und Erkennung von Rezidiven [23]. Die Computertomografie gilt trotz der damit verbundenen Strahlenbelastung als bildgebendes Verfahren der Wahl, da mit ihr eine optimale Darstellung von Knochenund Weichteilstrukturen möglich ist (s. auch ope- rative Therapie, Abb. 1-3) [1]. Die Magnetresonanztomografie ist dem CT hinsichtlich der Weichteildarstellung überlegen, liefert aber unterlegene Aussagen über die ossären Strukturen. Das konventionelle Röntgen der Nasennebenhöhlen wird zur Diagnostik der Rhinosinusitis generell nicht empfohlen [23].

Weitere wichtige diagnostische Bausteine für die Abklärung einer CRS sind Endoskopie und Computertomografie.

\section{Therapie}

$\nabla$

Die Therapieformen der CRS lassen sich in nichtoperative und operative Verfahren einteilen. Ziel jeder Therapie ist eine möglichst optimale Symptomkontrolle sowie die Vermeidung von Komplikationen. Grundsätzlich kommt eine Operation in der Regel nur dann in Frage, wenn die nicht-operativen Behandlungsverfahren ausgeschöpft sind.

\section{Nicht-operative Therapie}

Topische Glukokortikosteroide (GKS)

Das Ausmaß der Entzündungsreaktion bei CRS lässt sich durch die Anwendung von nasalen (topischen) GKS minimieren. Die Effekte sind bei der CRSwNP deutlicher belegt als bei der CRSsNP und lassen sich über die effektive Unterdrückung der eosinophilenassoziierten Entzündungsreaktion bei CRSwNP erklären $[1,23]$. Neuere Präparate, wie z.B. Mometason-furoat, haben eine geringe systemische Bioverfügbarkeit und damit wenig unerwünschte Wirkungen [32]. Ein erhöhtes Frakturrisiko wie bei Patienten mit COPD und inhalativer GKS-Therapie wurde ebenfalls nicht nachgewiesen [33]. Die unerwünschten Wirkungen bestehen in erster Linie in trockenen Nasenschleimhäuten und Epistaxis.

Die Dauer der Therapie hängt von der zugrundeliegenden Form einer CRS ab: bei unbehandelten Nasenpolypen und als Behandlungsversuch zur Vermeidung einer Operation sowie zur Rezidivprophylaxe nach Operation wird eine Therapie über 6 bis 12 Monate empfohlen [23]. Der Einsatz von topischen GKS im Rahmen einer akuten Exazerbation der CRS (AE-CRS) ist nicht klar definiert. Grundsätzlich empfiehlt das europäische Positionspapier AE-CRS wie eine akute Rhinosinusitis zu therapieren [1]. Topische GKS werden auch zur Behandlung einer akuten Rhinosinusitis empfohlen, allerdings nicht bei Vorliegen einer schweren und damit wahrscheinlich bakteriellen akuten Form [23].

\section{Systemische GKS-Therapie}

Die Wirksamkeit von systemischen GKS in der CRS-Therapie ist gut belegt, auch wenn zahlreiche Studien ihre Anwendung in Kombination mit topischen GKS und Antibiotika untersucht haben 
[1]. Insbesondere eine dauerhafte systemische GKS-Therapie sollte angesichts der potenziellen unerwünschten Wirkungen vermieden werden.

Topische GKS sind ein fester Bestandteil in der Therapie der CRS.

\section{Antibiotika}

Eine Bedeutung kommt ihnen vor allem in der Behandlung der AE-CRS zu. Die akute Exazerbation wird als Verschlechterung der vorbestehenden Beschwerden definiert und orientiert sich-ebenso wie die Behandlung - an der akuten Rhinosinusitis [1]. Die Basis der meisten akuten Exazerbationen wird am ehesten in viralen Infektionen gesehen [1]. Eine weitere Ursache könnte die repetitive Freisetzung von Bakterien aus Biofilmen sein [1].

Man geht davon aus, dass bei AE-CRS dieselben Erreger eine Rolle spielen wie bei der akuten Rhinosinusitis. Hierbei handelt es sich um Pneumokokken, Haemophilus influenzae, Staphylococcus aureus, Streptococcus pyogenes und Moraxella catarrhalis.

Die Indikationen zur antibiotischen Therapie sind in $\odot$ Tab. 2 aufgeführt.

Spezifische Angaben zur Therapiedauer in der akuten Exazerbation fehlen, sodass davon ausgegangen werden muss, dass sie den vorgeschlagenen Therapiedauern bei der akuten Rhinosinusitis entsprechen.

Die in Frage kommenden Präparate zeigt $\bullet$ Tab.3. Die empfohlene Therapiedauer ist in Klammern angegeben.

Bei gehäuften AE-CRS sollten Antibiotika präferiert werden, die unter „schwere Formen“ aufgeführt sind. Bei häufig rezidivierenden Episoden einer AE-CRS sollte über eine Keimgewinnung zur mikrobiologischen Diagnostik nachgedacht werden. Goldstandard hierfür ist immer noch die Punktion der Kieferhöhle [23].

Der Effekt einer antibiotischen Therapie ist vor allem in einer Symptomlinderung zu sehen. Darüber hinaus können sie eine Verkürzung der Krankheitsdauer um ein bis zwei Tage bewirken und damit Ausfallzeiten im Berufsleben verkürzen.

Die Wirksamkeit von Antibiotika in der Langzeittherapie der CRS ist schwieriger zu beurteilen, da die vorliegenden Studien unterschiedliche Definitionen von CRS verwendet haben [23]. Prinzipiell ist der Einsatz derselben Antibiotika möglich, die bei der AE-CRS zum Einsatz kommen. In Analogie zur Behandlung der COPD werden den Makroliden (in geringer Dosierung) in der CRS-Therapie auch antiinflammatorische Effekte zugeschrieben. Wichtig ist in diesem Kontext auf die aus der COPD-Therapie bekannten Limitationen bei kardial vorerkrankten Patienten zu achten, die sich aus einer Untersuchung zum kardiovaskulären Risikoprofil von Azithromycin in der Kurzzeit-
Tab.2 Indikationen zur antibiotischen Therapie bei AE-CRS (in Anlehnung an [23]).

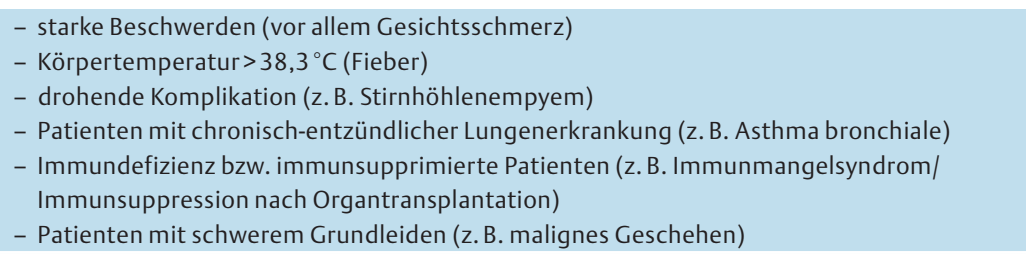

Tab.3 Antibiotika, die zur Behandlung der AE-CRS in Frage kommen (in Anlehnung an [23]).

\begin{tabular}{|c|c|}
\hline \multicolumn{2}{|c|}{ Antibiotische Therapie der akuten Exazerbation einer CRS } \\
\hline Mittel der Wahl: & Amoxicillin (7 Tage) \\
\hline Alternativen: & $\begin{array}{l}\text { Aminopenicillin mit } \beta \text {-Laktamaseinhibitor (7 Tage) } \\
\text { (orales Cephalosporin der Gruppe } 2 \text { - auf Grund der geringen Wirk- } \\
\text { spiegel bei Darreichungsform per os aus unserer Sicht zu meiden) } \\
\text { Makrolid (z. B. Azithromycin - } 3 \text { Tage) } \\
\text { Cotrimoxazol (7 Tage) } \\
\text { Clindamycin (mit allerdings schmalem Wirkspektrum - } 7 \text { Tage) } \\
\text { Doxycyclin (7 Tage) }\end{array}$ \\
\hline \multicolumn{2}{|c|}{ Schwere Formen (Risikofaktoren) } \\
\hline Mittel der Wahl: & $\begin{array}{l}\text { Aminopenicillin mit } \beta \text {-Laktamaseinhibitor (7 Tage) } \\
\text { (orales Cephalosporin der Gruppe 2, Cefotaxim - s. o.) }\end{array}$ \\
\hline Alternativen: & $\begin{array}{l}\text { (orales Cephalosporin der Gruppe } 3 \text { - s. o.) } \\
\text { Ciprofloxacin (7 Tage) } \\
\text { Levofloxacin, Moxifloxacin (jeweils } 5 \text { Tage) }\end{array}$ \\
\hline
\end{tabular}

anwendung ergeben haben [34]. Die besten Ergebnisse erzielt die niedrigdosierte MakrolidLangzeittherapie nach jetzigem Kenntnisstand bei Patienten mit CRSsNP mit normalem SerumIgE-Spiegel über einen Zeitraum von 12 Wochen [1].

Kurzzeitige Therapien, definiert als Zeiträume unter 3 Wochen, sind von geringer Bedeutung. In einer Studie über 3 Wochen mit Doxycyclin bei CRSwNP konnte lediglich ein Effekt auf die Polypengröße beobachtet werden [1]. Langfristige Therapien, definiert als Zeiträume über 4 Wochen, können in Kombination mit GKS als Alternative zur operativen Therapie erwogen werden [23]. Für die CRSsNP gelten sie als Therapieoption, wenn Kochsalzspülungen und topische GKS nicht zu einer ausreichenden Symptomkontrolle führen [1]. Zu berücksichtigen sind die üblichen unerwünschten Wirkungen von antibiotischen Therapien, wie z.B. Resistenzentwicklung, Begünstigung von Mykosen, Allergien/Unverträglichkeiten und gastrointestinale Probleme.

Studien zur Lokalanwendung mit Mupirocin-Nasensalbe konnten keinen anhaltenden Therapieeffekt belegen [35,36], sodass keine Empfehlung ausgesprochen werden kann [1].

Antibiotika haben vor allem eine Bedeutung in der Behandlung der AE-CRS.

\section{Leukotrienantagonisten}

Die Wirksamkeit von Leukotrienantagonisten bei der CRSwNP wurde in mehreren Studien überprüft. Mit einer Dosis von $10 \mathrm{mg}$ Montelukast pro

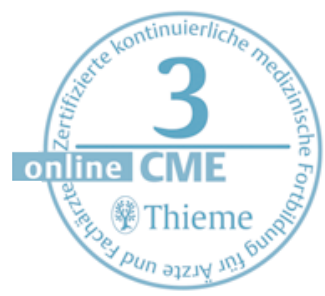


Tag zeigten sich sowohl prä- als auch postoperativ positive Effekte hinsichtlich der eosinophilen Entzündungsreaktion, klinischer SymptomenScores, der nasalen Belüftung sowie der Nasensekretion [37]. Montelukast wurde als add-onTherapie zu GKS oder alternativ zu topischen GKS untersucht [38]. Die Behandlungszeiträume lagen im Bereich von 6 Wochen bis zu einem Jahr [1]. Ein Therapieversuch sollte zunächst 6 Wochen bis 3 Monate umfassen.

Wichtigster Vorteil einer Montelukasttherapie sollte die Senkung der postoperativen Rezidivrate sein, die trotz des konsequenten Einsatzes von topischen GKS bei diesem Patientenkollektiv noch immer im Bereich von 3 bis 10\% liegt.

Eine systematische Untersuchung zum Einsatz von Montelukast an großen CRS-Patientenkollektiven steht aus und sollte zügig erfolgen.

Auf die Beziehung zwischen einer MontelukastBehandlung und dem Entstehen eines CSS sei an dieser Stelle erneut hingewiesen.

\section{Antihistaminika/Spezifische (subkutane) Immuntherapie (S(C)IT)}

Der Einsatz von Antihistaminika ist auf die Anwendung bei CRS-Patienten beschränkt, die eine gesicherte allergische Rhinitis mit entsprechenden Beschwerden haben. Der Effekt der SCIT wurde bei Patienten mit allergischer Pilzrhinosinusitis überprüft. Eine Langzeitwirksamkeit konnte nicht belegt werden [1].

\section{Adaptive Desaktivierung}

Patienten mit Aspirin ${ }^{\circledR}$-sensitivem Asthma sprechen schlechter auf eine NNH-Operation an. Eine adaptative Desaktivierung und Erhaltungstherapie mit Acetylsalicylsäure kann Beschwerden im Bereich der oberen Atemwege lindern und die Rezidivrate von Polypen minimieren [1]. Sie wird daher ergänzend $\mathrm{zu}$ den sonstigen etablierten nicht-operativen und operativen Verfahren empfohlen, sollte aber nur in erfahrenen Zentren erfolgen.

Zur Schmerztherapie können Cyclooxygenase (COX)-2-Hemmer eingesetzt werden, da diese eine gute Verträglichkeit gezeigt haben [1].

\section{Topische Therapien}

Nasenspülungen/-sprays mit hypertonen gepufferten Lösungen werden bei CRS empfohlen, da sie eine Beschwerdelinderung bewirken können [23]. Selbiges gilt für die Anwendung warmer Dämpfe $\left(42-45^{\circ} \mathrm{C}\right)$. Eine Empfehlung für den Einsatz ätherischer Öle hingegen existiert nicht [23].

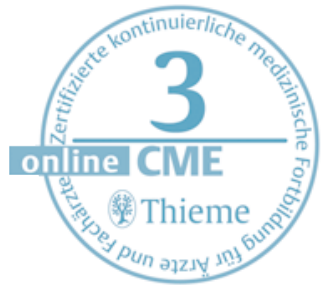

Es gibt keine Evidenz für die Wirksamkeit von Sekretolytika (z.B. N-Acetylcystein) und Dekongestiva. Letztere haben aber zumindest eine symptomatische Wirksamkeit. Darüber hinaus konnte in randomisierten kontrollierten Studien biotika und Phytotherapeutika in der CRS-Therapie belegt werden [1].

Für viele der häufig verwendeten therapeutischen Mittel (z. B. Sekretolytika, Dekongestiva) fehlt die Evidenz.

\section{Operative Therapie der CRS}

Indikationsstellung

Versagen die konservativen Therapieformen der CRS, so ist an eine operative Sanierung zu denken. Die Indikation für ein operatives Vorgehen sollte immer dann gestellt werden, wenn eine erhebliche Nasenatmungsbehinderung durch eine Polyposis nasi oder rezidivierende Infekte in der Anamnese zu eruieren sind. Die Diagnose einer CRS sollte sicher an den oben bereits genannten Kriterien gestellt worden sein. Eine konservative Therapie sollte ohne Erfolge geblieben oder aber die vorliegenden Befunde so ausgeprägt sein, dass medikamentös keine wesentliche Befundbesserung zu erwarten ist.

Streng einseitige chronische Befunde sind immer eine Operationsindikation. Diese können zum einen auf einer Aspergillose des NNH-Systems beruhen, können aber auch auf der Grundlage eines invertierten Papilloms [39] oder Malignoms [40] entstanden sein und sind somit zwingend einem operativen Vorgehen zuzuführen. Davon abzugrenzen sind chronisch einseitige Befunde bei entzündlichen Veränderungen des Kauapparates.

Ergänzende präoperative Diagnostik

Zur Planung eines operativen Eingriffs am NNHSystem ist eine sichere Diagnosestellung erforderlich. Dies sollte im Vorfeld mit Hilfe der bereits genannten Diagnostik erfolgt sein.

Unabdingbar für die Vorbereitung eines operativen Vorgehens ist die Anfertigung einer NNHComputertomografie. Dabei ist die Darstellung der knöchernen Landmarken besonders wichtig, um intraoperativ sicher und komplikationsarm arbeiten zu können. Bei der Akquisition einer Computertomografie der NNH sollte immer auf die Anwendung eines Niedrigdosisprotokolls geachtet werden, um die Strahlenexposition der Augenlinsen zu minimieren [41]. Eine konventionelle Röntgenaufnahme der NNH ist nicht ausreichend. Die Magnetresonanztomografie kann diagnostische Hinweise bieten, hat aber den Nachteil, dass sie wegen der unzureichenden Darstellung der knöchernen Strukturen als Operationsvorbereitung allein nicht nutzbar ist.

Vor operativer Sanierung eines streng einseitigen Befundes ist eine zahnärztliche Diagnostik erforderlich. Bei Symptomen der Augen sollte ein Ophthalmologe hinzugezogen werden. bzw. systematischen Reviews kein Vorteil für Pro-

\section{Operationstechniken}

In der NNH-Chirurgie kommen verschiedene Operationsverfahren zum Einsatz, die auch kombiniert werden können. Die endonasalen Techni- 
ken werden mit Hilfe des binokularen Operationsmikroskops und der starr endoskopischen Technik durchgeführt (Functional Endoscopic Sinus Surgery, FESS). Auch konventionelle Zugänge von außen kommen gelegentlich in der NNHChirurgie zum Einsatz. Dies betrift vor allem komplikative Verläufe der Stirnhöhle und des Siebbeins. Die Eröffnung der Kieferhöhle über den Mundvorhof (Operation nach Caldwell-Luc) ist heute für die Therapie der CRS obsolet.

Das binokulare Operationsmikroskop bietet dem Operateur konstruktionsbedingt den Vorteil, dass eine Tiefenabschätzung innerhalb der Nase ermöglicht wird. Gleichzeitig ist es frei schwebend fixiert, was dem Operierenden ein beidhändiges Arbeiten mit verschiedenen Instrumenten erlaubt. Nachteilig sind die geringe Tiefenschärfe und der Lichtabfall in der Tiefe. Das Mikroskop ermöglicht keine Kontrolle verdeckt gelegener Bereiche.

Die starr-endoskopische Technik ermöglicht dagegen mit verschiedenen Winkeloptiken, die Anatomie der NNH auch in schwer einsehbaren Anteilen zu beurteilen. Die Lichtsituation ist immer gleichbleibend gut. Anders als bei der Mikroskopie kann aber nicht beidhändig gearbeitet werden, da auch das Endoskop vom Operateur gehalten werden muss [42]. Blut und Sekret können das Endoskop verunreinigen und die Sicht behindern, sodass das Endoskop häufig gereinigt werden muss. Ein großer Nachteil der endoskopischen Methode ist die fehlende Dreidimensionalität, die eine Tiefenabschätzung erschwert.

Neueste Entwicklungen gehen aber hin zu elektronischen Kameraendoskopen, die über zwei getrennte Chips ein dreidimensionales Bild liefern können [43].

Sowohl die mikroskopische als auch die endoskopische Technik werden gerne in Kombination mit einem computergestützten Navigationssystem genutzt, welches zusätzliche Sicherheit bei der Identifikation von Landmarken, insbesondere bei komplexen Fällen, bieten kann [42]. In der klinischen Praxis werden Mikroskop und Endoskop oft auch in Kombination verwendet.

Offen chirurgische Techniken sind bei der Chirurgie der CRS nur selten anzuwenden. Komplexe Verläufe insbesondere der Stirnhöhle können dies gelegentlich erforderlich machen. Offen chirurgische Techniken kommen vor allem bei der Therapie von Malignomen der Nasenhaupt- und -nebenhöhlen zur Anwendung.

Die heutige operative Therapie der NNH wird in der Regel endonasal und endoskopisch durchgeführt.

\section{Operative Vorgehensweise}

Ziel der operativen Therapie einer CRS ist die Entfernung entzündlich veränderter Schleimhautareale sowie eine Verbesserung der gesamten Belüftungs- und Drainagesituation des Gesamtsys- tems. Wichtigster Zugangsweg ist dabei der mittlere Nasengang, in den die meisten Sinus paranasales direkt oder indirekt drainieren.

Die Operationen an den NNH bauen entsprechend der Anatomie aufeinander auf. Der einfachste Eingriff ist die Infundibulotomie, mit der durch Entfernen des leicht federnden Processus uncinatus an der lateralen Nasenwand die Sicht auf das vordere Siebbein mit der Bulla ethmoidales und das Kieferhöhlenostium freigegeben wird [44] (৫ Abb.4).

Der Eingriff kann von hier aus zunächst zu einer isolierten Kieferhöhlensanierung ausgedehnt werden. Dabei wird das natürliche Kieferhöhlenostium vorsichtig erweitert. Dabei sind genaue anatomische Kenntnisse über den Verlauf des Tränennasenganges erforderlich. Fensterungen der Kieferhöhle über den unteren Nasengang werden heute nicht mehr durchgeführt, da ein solch artifizielles Ostium nicht im physiologischen Zilientransportweg der Kieferhöhlenschleimhaut liegt. Bei weiter reichenden Problemen erfolgt daran anschließend das Eröffnen der vordersten Siebbeinzelle, der Bulla ethmoidalis. Dieser Eingriff wird anteriore Ethmoidektomie genannt. Die typische Landmarke ist der maxilloethmoidale Winkel, dem Übergang von Kieferhöhle zu eröffnetem Siebbein. Innerhalb des Siebbeins finden sich ausgesprochen dünnwandige Zellen, die durch vorsichtigen Druck des Instrumentes eröffnet werden können und dann unter ständiger Sicht abgetragen werden. Die laterale Begrenzung dieses Operationsgebietes stellt die Lamina papyracea dar, die das Siebbein von der Augenhöhle trennt. Medial ist das Operationsfeld durch die mittlere Muschel begrenzt.

Ausgehend von diesem Operationssitus kann auch der kranial gelegene Recessus frontalis als Zugang zur Stirnhöhle erreicht werden und bei Beteiligung der Stirnhöhlenschleimhaut ebenfalls zur besseren Drainage und Belüftung erweitert werden.

Ist eine vordere Siebbeinausräumung nicht ausreichend, so werden die Siebbeinzellen weiter nach hinten ausgeräumt, bis die Schädelbasis erkennbar ist.

Wurde das gesamte Siebbein von Zellen befreit, hat der Operateur die Möglichkeit, vom Siebbein aus das Keilbein zu eröffnen. Hierfür steht allerdings auch ein alternativer, isolierter Zugangsweg zur Verfügung, bei dem das natürliche Ostium des Sinus sphenoidalis in der Verlängerung des Septums aufgesucht wird [45].

In der Revisionschirurgie ist die Zielsetzung einer Verbesserung von Belüftung und Drainage weiterhin das vorrangige Ziel. Ebenso wie bei Primäreingriffen werden krankhafte Schleimhautareale und störende Hindernisse entfernt. Die Chirurgie einer erneut aufgetretenen CRS ist im Gegensatz zu Ersteingriffen aber deutlich erschwert, da die klassischen Landmarken oft bereits reseziert wurden.

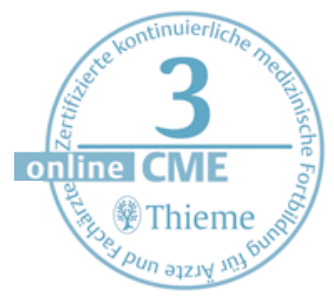


Abb.4 Schemazeichnung des NNH-Systems. Der Schlüssel zum operativen Vorgehen ist der Processus uncinatus, der - nach Abtragung - den Weg zu allen Nasennebenhöhlen frei gibt. Die farblich verschiedenen Gruppen können einzeln oder in Kombination operativ saniert werden.

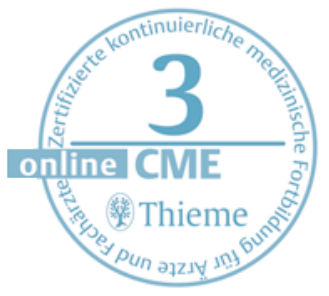

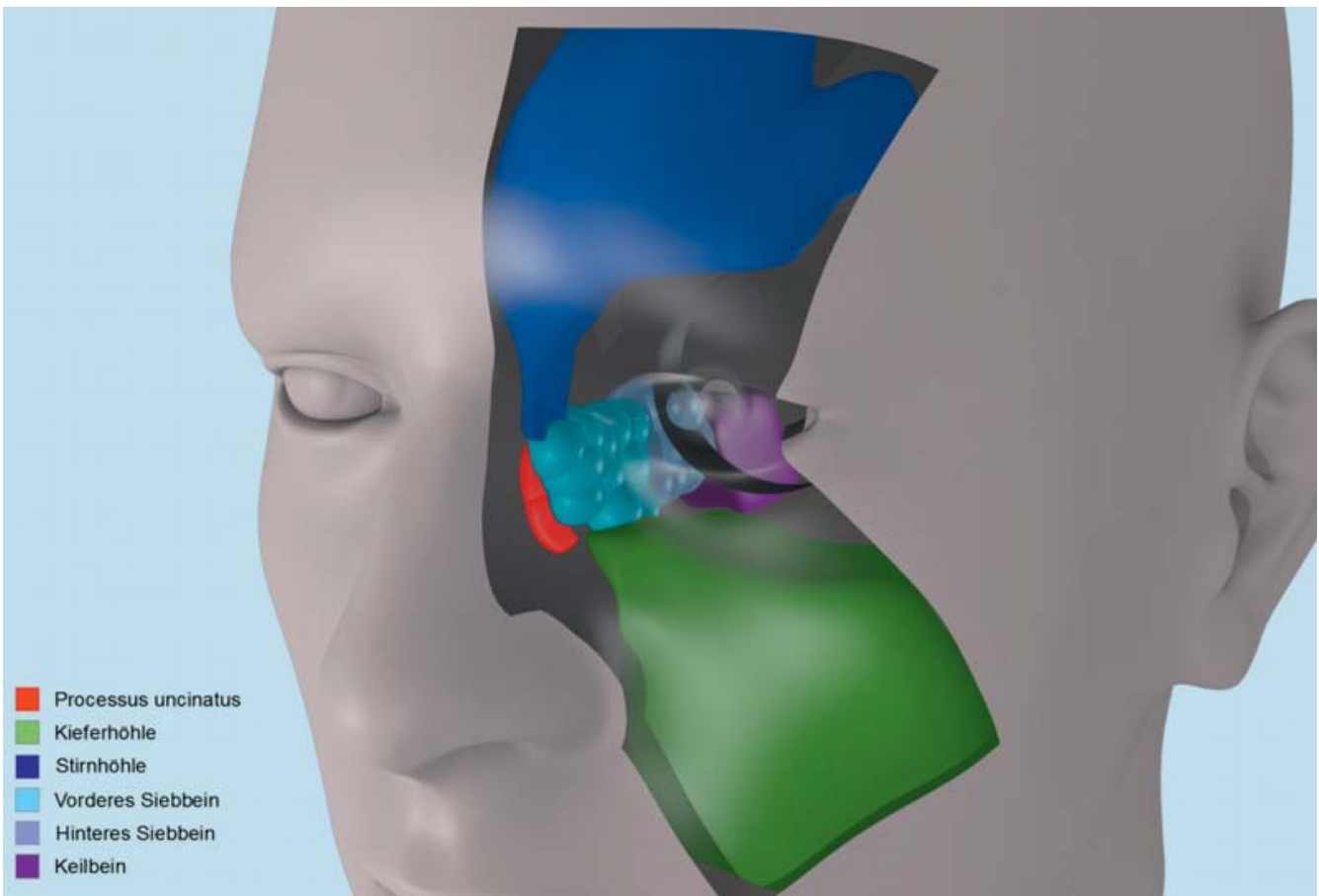

Mit der operativen Therapie soll die Belüftungssituation im Bereich der NNH verbessert und entzündlich veränderte Schleimhautareale entfernt werden.

\section{Komplikationsmöglichkeiten von NNH-Eingriffen}

Die anatomischen Landmarken der NNH-Chirurgie bestehen zu großen Anteilen aus wichtigen Strukturen, deren Verletzung erhebliche Folgen für den Patienten haben können.

Die enge topografische Beziehung des Siebbeins zum Auge erfordert es, den Patienten bereits im Vorfeld über Augenhämatome und Doppelbilder bei Augenmuskelschädigungen aufzuklären. Bei arteriellen Blutungen in die Augenhöhle kann es innerhalb kürzester Zeit zu erheblichen Kompressionssymptomen kommen, die zur Erblindung des betreffenden Auges führen können. In einem solchen Fall sind dekomprimierende, operative Maßnahmen erforderlich.

Im Rahmen der Kieferhöhlenchirurgie wurde bereits auf die Möglichkeit von Verletzungen des Tränengangsystems hingewiesen. Eine weitere wichtige Komplikationsmöglichkeit der Siebbeinchirurgie sind Perforationen der Schädelbasis. Tritt ein derartiges Problem intraoperativ auf, so ist die entstandene Liquorfistel mehrschichtig zu decken. Geschieht dies nicht oder bleibt die Schädelbasisperforation zunächst unbemerkt, so ist eine ständige Rhinoliquorrhoe zu erwarten. Für den Patienten erhöht sich dann das Risiko, an einer Meningitis als Spätfolge im weiteren Verlauf seines Lebens zu erkranken [46]. Größere Schädelbasisdurchtritte mit unbeabsichtigter Resektion von Hirngewebe sind ebenfalls möglich, aber ausgesprochen selten.
Ein besonderes Problem der Keilbeinhöhleneingriffe, aber auch des hinteren Siebbeins können Verletzungen des Nervus opticus sein. Neben dieser schwerwiegenden Komplikation besteht innerhalb der Keilbeinhöhle zusätzlich noch das Risiko von Schädigungen der Arteria carotis interna, die ebenfalls, nur durch eine dünne knöcherne Lamelle bedeckt, die laterale Wand des Sphenoidalsinus passiert.

Langfristig können dauerhafte Riechstörungen, endonasale Borkenbildungen, Schmerzsyndrome und narbige Verwachsungen zu einer Belastung für den operierten Patienten werden [47]. Eine Übersicht über mögliche Komplikationen der operativen Therapie gibt $\bullet$ Tab. 4 . rapie erklären sich über lokale Probleme im Bereich der Nase/ NNH sowie die topografische Nähe zu Auge und Schädelbasis.

\section{Postoperative Maßnahmen}

Bei endonasalen Eingriffen kann es leicht zu postoperativen Blutungen kommen. Um dies zu verhindern, aber auch um die erarbeiteten Strukturen des NNH-Systems räumlich zu erhalten, werden üblicherweise für zwei Tage Fingerlingstamponaden eingesetzt.

Nach deren Entfernung wird mit der postoperativen Nachsorge begonnen. Dazu werden nach Aufweichen durch Inhalationen Nasensekrete und alte Blutreste unter Sicht, teils auch endoskopisch, abgesaugt. Bei der regelmäßigen Reinigung der Nase werden größere Krusten vorsichtig entfernt. Ziel dieser Maßnahmen ist der Erhalt der Belüftung und das Verhindern von Verwachsungen.
Die möglichen Komplikationen einer operativen The- 
Mit fortschreitender Wundheilung wird die anfänglich mehrfach täglich erforderliche Maßnahme mehr und mehr reduziert, bis nach etwa drei Wochen keine regelmäßigen Absaugvorgänge mehr erforderlich sind.

Begleitend sollte der Patient sich selbst mit Xylomethazolin-haltigem Nasenspray behandeln und die entstehenden Verkrustungen mit einem Nasenöl weich erhalten. Zur Pflege des Vestibulum nasi hat sich eine weiche fetthaltige Salbe etabliert. Zwei Wochen postoperativ können aber auch zusätzlich Nasenspülungen durchgeführt werden.

Zur Rezidivprophylaxe hat sich die Gabe eines topischen Glukokortikosteroids als weitere begleitende Maßnahme durchgesetzt. Es gibt Hinwiese, dass sich eine solche Therapie auch positiv auf die Wundheilung auswirken könnte [48]. In Fällen mit einem erhöhten Risiko für eine Rezidiverkrankung kann begleitend auch eine systemische GKS-Therapie empfohlen werden. Rezidive nach FESS lassen sich möglicherweise durch ein besonders hohes Ausmaß an Entzündungsreaktion erklären (inflammatory load hypothesis) [49]. Insgesamt sind Rezidive nach NNH-OP mit etwa $20 \%$ der operierten Fälle als häufig zu bewerten.

Die operative Therapie kann nicht nur zur Linderung der Beschwerden durch die CRS führen. Sie kann bei Patienten mit Asthma bronchiale und CRSwNP oder CRSsNP zur Verbesserung der Asthmakontrolle führen [1].

Postoperativ ist eine intensive Nasenpflege - teils auch endoskopisch - sowie der frühzeitige Einsatz von topischen GKS wichtig.

\section{Eingriffe in Oberflächenanästhesie}

Die Nase ist mit ihrer guten Erreichbarkeit auch für Eingriffe in Oberflächenanästhesie geeignet. Für die Durchführung von Nasennebenhöhleneingriffen beschränkt sich dies aber nur auf den Teil der einfachen Eingriffe. Trotz Anästhesie können diese sehr schmerzhaft sein. Daher werden sie auch nur sehr selten durchgeführt und spielen in der klinischen Praxis keine große Rolle.

Bei einer ausgeprägten Polyposis nasi kann eine einfache Polypektomie indiziert sein, die dann auch in Oberflächenbetäubung durchgeführt werden kann. Dieser Eingriff ist aber im strengen Sinne der Nasenhaupthöhle zuzuordnen, auch wenn die zugrundeliegende Erkrankung eine CRS ist. Dabei wird mit einer Schlinge der Polyp erfasst und unter vorsichtigem Zug abgeschnürt und entfernt.

In der Folge dieses kleinen ambulanten Eingriffs ist der Patient sofort wieder in der Lage, ausreichend gut durch die Nase Luft zu holen. Leider ist zu erwarten, dass die Polyposis innerhalb weniger Wochen bis Monate wieder auftritt und der Eingriff wiederholt werden muss. Daher ist die einfache Polypektomie nur dann zu empfehlen,
Tab.4 Mögliche Komplikationen eines NNH-Eingriffs.

\begin{tabular}{l}
$\begin{array}{l}\text { Mögliche Komplikationen eines Nasennebenhöhlen- } \\
\text { eingriffs }\end{array}$ \\
\hline Orbitale Hämatome \\
Orbitale Kompressionen \\
Augenmuskelschädigungen \\
Augenverletzungen \\
\hline Schädigungen des Tränenganges \\
\hline Rhinoliquorrhoe \\
Meningitis \\
Encephalitis \\
Hirnverletzungen \\
\hline Schädigung des Nervus opticus \\
Verletzung der Arteria carotis interna \\
\hline Anosmien \\
Borkenbildungen \\
Narbige Stenosen
\end{tabular}

wenn ein weiteres operatives Vorgehen auf Grund von Kontraindikationen nicht durchgeführt werden kann.

Eingriffe in Oberflächenanästhesie sind die Ausnahme und dienen vor allem der Polypektomie einzelner Polypen.

\section{Fazit}

Ziel der CRS-Therapie ist die Kontrolle der Entzündungsreaktion, die sich klinisch in einer Symptomkontrolle widerspiegeln sollte. Patienten mit CRS sollten hierzu unabhängig von der CRS-Form mit einem topischen GKS behandelt werden. Systemische GKS sind auf Grund ihres Profils an unerwünschten Wirkungen nach Möglichkeit zu meiden. Die antibiotische Therapie hat ihren Stellenwert vor allem in der Behandlung von akuten Exazerbationen. Treten diese gehäuft auf, sollte auch an ein Immunmangelsyndrom gedacht werden. Bei einer bakteriell verursachten AE-CRS ist die Anwendung von topischen GKS restriktiv zu sehen. Bei Versagen der konservativen Therapiemöglichkeiten kommen chirurgische Verfahren in Frage. Hier ist vor allem die FESS zu nennen. Die postoperative Rezidivrate ist insgesamt hoch, sodass im Laufe eines Lebens weitere operative Eingriffe erforderlich werden können. Zur Rezidivprophylaxe sollten wiederum topische GKS zum Einsatz kommen. 
33-jähriger Pat. mit CRSwNP, therapierefraktär unter einjähriger Dauertherapie mit Mometason-furoat und teilweise additiv Prednisolon systemisch in einer Dosierung zwischen 25-50 mg einmal täglich über Zeiträume von jeweils einer Woche. Ferner gehäufte antibiotische Therapien mit Cefaclor, Sultamicillin, Doxycyclin, Levofloxacin sowie Moxifloxacin bei AE-CRS (im Schnitt alle 4 Wochen). Vorbekannter gastroösophagealer und laryngopharyngealer Reflux, therapiert mit Esomeprazol $40 \mathrm{mg}$ ein- bis zweimal täglich. Darüber hinaus saisonale allergische Rhinokonjunktivitis mit polyvalenten Sensibilisierungen gegen Birken-, Gräser- und Roggen-

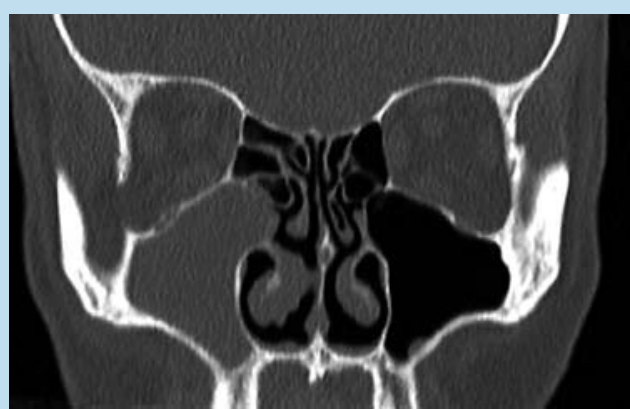

Abb.5 Ausgedehnte Schleimhautschwellung und vollständige Verlegung des rechten Sinus maxillaris. Geringe Schleimhautschwellung auch am Boden des linken Sinus maxillaris und im Nasenbinnenraum.

\section{Interessenkonflikt}

$\nabla$

Die Autoren geben an, dass kein Interessenkonflikt besteht.

\section{Institute}

${ }^{1}$ Thoraxzentrum Ruhrgebiet, Klinik für Pneumologie und Infektiologie, EVK Herne - Betriebsstelle Eickel (Chefarzt: Prof. Dr. med. S. Ewig)

2 Berufsgenossenschaftliches Universitätsklinikum Bergmannsheil, Medizinische Klinik III - Pneumologie, Allergologie, Schlaf- und Beatmungsmedizin (Direktor: Prof. Dr. med. J. Behr)

3 Universitätsklinik für Hals-, Nasen- und Ohrenheilkunde, Kopf- und Halschirurgie der Ruhr-Universität Bochum am St. Elisabeth Hospital (Direktor: Prof. Dr. med. S. Dazert) ${ }^{4}$ Institut für Diagnostische und Interventionelle Radiologie und Nuklearmedizin, Berufsgenossenschaftliches Universitätsklinikum Bergmannsheil GmbH (Direktor: Prof. Dr. med. V. Nicolas)

${ }^{5}$ Institut für Pathologie der Ruhr-Universität Bochum am Berufsgenossenschaftlichen Universitätsklinikum Bergmannsheil (Direktorin: Prof. Dr. med. A. Tannapfel) pollen, behandelt in den entsprechenden Zeiträumen mit Ketotifen Augentropfen und Desloratadin $5 \mathrm{mg}$ einmal täglich per os. Hinweise für eine Analgetika-Intoleranz bzw. ein Asthma bronchiale fanden sich. Auf Grund des Verlaufs mit zahlreichen AE-CRSs und hohem GKS-Einsatz fiel die Entscheidung zur FESS. Präoperativ wurde ein CT der NNH angefertigt ( $\bullet$ Abb.5). Intraoperativ wurden Schleimhautproben aus der rechten Kieferhöhle und den Siebbeinzellen gewonnen ( Abb.6). Ein Jahr nach der FESS hat sich die Belüftungssituation deutlich verbessert. Die Exazerbationsrate ist gesunken.

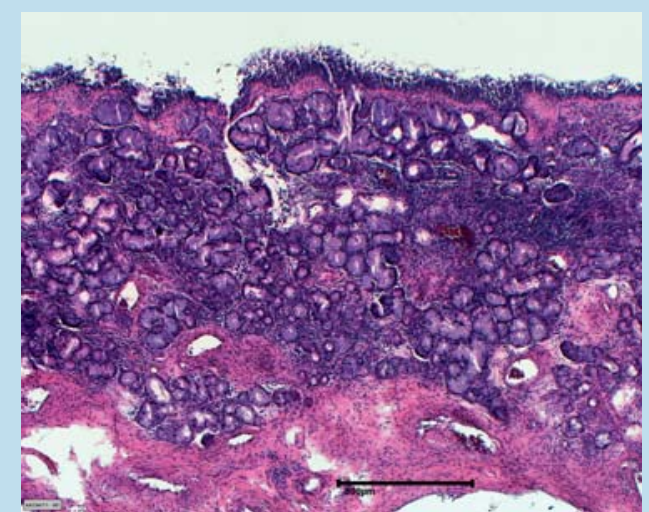

Abb. 6 Hyperplastische Schleimhaut mit einer chronischen Entzündung und einer deutlichen Hyperplasie der mukoiden Drüsen und polypöser Schleimhaut, Hämatoxylin-Eosin-Färbung, Maßstab im Bild.

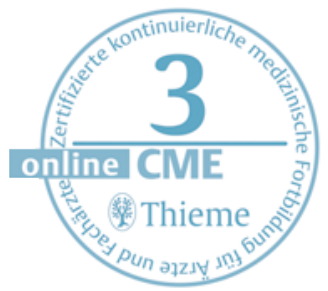

\section{Literatur}

1 Fokkens WJ, Lung VJ, Mullol J et al. European Position Paper on rhinosinusitis and nasal polyps 2012. Rhinol Suppl 2012; 23: 1-298

2 Hastan D, Fokkens WJ, Bachert C et al. Chronic rhinosinusitis in Europe - an undererstimated disease. A GA2LEN study. Allergy 2011; 66: 1216-1223

3 Kardos P, Berck H, Fuchs KH et al. Leitlinie der Deutschen Gesellschaft für Pneumologie und Beatmungsmedizin zur Diagnostik und Therapie von erwachsenen Patienten mit akutem und chronischem Husten. Pneumologie 2010; 64: 336-373

4 Poelmans J, Tack J. Extraesophageal manifestations of gastrooesophageal reflux. Gut 2005; 54: $1492-1499$

5 Koufman JA, Aviv JE, Casiano RR et al. Laryngopharyngeal reflux: position statement of the committee on speech, voice, and swallowing disorders of the American Academy of Otolaryngology-Head and Neck Surgery. Otolaryngol Head Neck Surg 2002; 127: 32-35

6 Masi AT, Hunder GG, Lie JT et al. The American College of Rheumatology 1990 criteria for the classification of Churg-Strauss syndrome (allergic granulomatosis and angiitis). Arthritis Rheum 1990; 33: 1094-1100

7 Jennette JC, Falk RJ, Andrassy K et al. Nomenclature of systemic vasculitides. Proposal of an international consensus conference. Arthritis Rheum 1994; 37: 187- 192

8 Luqmani RA, Suppiah R, Grayson PC et al. Nomenclature and classification of vasculitis - update on the ACR/EULAR diagnosis and classification of vasculitis study (DCVAS). Clin Exp Immunol 2011; 164 (Suppl. 01): 11 13 
9 Solans R, Bosch JA, Selva A et al. Montelukast and ChurgStrauss syndrome. Thorax 2002; 57: 183-185

10 Stevenson DD, Hankammer MA, Mathison DA et al. Aspirin desensitization treatment of aspirin-sensitive patients with rhinosinusitis-asthma: long-term outcomes. J Allergy Clin Immunol 1996; 98: 751 - 758

11 Jarvis D, Newson R, Lotvall J et al. Asthma in adults and its association with chronic rhinosinusitis: the GA2LEN survey in Europe. Allergy 2012; 67: 91 - 98

12 Balkissoon R, Kenn K. Asthma: Vocal cord dysfunction (VCD) and other dysfunctional breathing disorders. Semin Respir Crit Care Med 2012; 33: 595-605

13 Davidson TM, Murphy C, Mitchell M et al. Management of chronic sinusitis in cystic fibrosis. Laryngoscope 1995; 105: $354-358$

14 Vital D, Hofer M, Boehler A et al. Posttransplant sinus surgery in lung transplant recipients with cystic fibrosis: a single institutional experience. Eur Arch Otorhinolaryngol 2012: epub ahead of print

15 Ramos RT, Salles C, Gregório PB et al. Evaluation of the upper airway in children and adolescents with cystic fibrosis and obstructive sleep apnea syndrome. Int J Pediatr Otorhinolaryngol 2009; 73: $1780-1785$

16 Ramsey BW, Davies J, McElvaney NG et al. A CFTR potentiator in patients with cystic fibrosis and the G551D mutation. N Engl J Med 2011; 365: $1663-1672$

17 Reed J, deShazo RD, Houle TT et al. Clinical features of sarcoid rhinosinusitis. Am J Med 2010; 123: 856-862

18 deShazo RD, Stringer SP. Atrophic rhinosinusitis: progress toward explanation of an unsolved medical mystery. Curr Opin Allergy Clin Immunol 2011; 11: 1 - 7

19 Agrafiotis M, Vardakas $K Z$, Gkegkes ID et al. Ventilatorassociated sinusitis in adults: systematic review and meta-analysis. Respir Med 2012; 106: 1082 - 1095

20 Holzapfel L, Chevret S, Madinier G et al. Incidence of long term oro- or nasotracheal intubation on nosocomial maxillary sinusitis and pneumonia: results of a randomized clinical trial (300 patients). Crit Care Med 1993; 21 $1132-1138$

21 Dalhoff $K$, Abele-Horn M, Andreas S et al. Epidemiologie, Diagnostik und Therapie erwachsener Patienten mit nosokomialer Pneumonie. Pneumologie 2012; 66: 707 765

22 Verse T. Nebenwirkungen der nasalen CPAP-Ventilationstherapie. HNO 2000; 48: 706-715

23 Stuck BA, Bachert C, Federspil $P$ et al. Leitlinie „Rhinosinusitis“ - Langfassung. HNO 2012; 60: 141-162

24 Benninghoff A. Anatomie. Makroskopische Anatomie, Embryologie und Histologie des Menschen - Band I. München, Wien, Baltimore: Urban \& Schwarzenberg; 1994: $529-541$

25 Lombardi C, Passalacqua G, Canonica GW. The functional connection between oral allergy syndrome and united airways disease assessed by oral challenge. Ann Allergy Asthma Immunol 2012; 108: 30-33

26 Oztürk AB, Damadoglu E, Karakaya G et al. Does nasal hair (vibrissae) density affect the risk of developing asthma in patients with seasonal rhinitis? Int Arch Allergy Immunol 2011; 156: 75-80

27 Tatar EC, Tatar I, Ocal B et al. Prevalence of biofilms and their response to medical treatment in chronic rhinosinusitis without polyps. Otolaryngol Head Neck Surg 2012; 146: $669-675$

28 Tan NC, Foreman A, Jardeleza C et al. The multiplicity of Staphylococcus aureus in chronic rhinosinusitis: correlating surface biofilm and intracellular residence. Laryngoscope 2012; 122: 1655-1660

29 Foreman A, Holtappels G, Psaltis A/ et al. Adaptive immune responses in Staphylococcus aureus biofilm-associated chronic rhinosinusitis. Allergy 2011; 66: 1449-1456
30 Tomassen P, Van Zele T, Zhang N et al. Pathophysiology of Chronic Rhinosinusitis. Proc Am Thorac Soc 2011; 8 : $115-120$

31 Reh DD, Lin SY, Clipp SL et al. Secondhand tobacco smoke exposure and chronic rhinosinusitis: a population-based case-control study. Am J Rhinol Allergy 2009; 23: 562 567

32 Mösges R, Bachert C, Rudack C et al. Efficacy and safety of mometasone furoate nasal spray in the treatment of chronic rhinosinusitis. Adv Ther 2011; 28: 238-249

33 Loke YK, Cavallazzi R, Singh S. Risk of fractures with inhaled corticosteroids in COPD: systematic review and meta-analysis of randomised controlled trials and observational studies. Thorax 2011; 66: 699-708

34 Ray WA, Murray KT, Hall $K$ et al. Azithromycin and the risk of cardiovascular death. N Engl J Med 2012; 366: 1881 1890

35 Jervis-Bardy J, Boase S, Psaltis A et al. A randomized trial of mupirocin sinonasal rinses versus saline in surgically recalcitrant staphylococcal chronic rhinosinusitis. Laryngoscope 2012; 122: 2148-2153

36 Jervis-Bardy J, Wormald PJ. Microbiological outcomes following mupirocin nasal washes for symptomatic, Staphylococcus aureus-positive chronic rhinosinusitis following endoscopic sinus surgery. Int Forum Allergy Rhino 2012; 2: 111 - 115

37 Rasp G. Is there a role for leukotriene antagonists in the prevention of recurrent nasal polyps? Curr Opin Allergy Clin Immunol 2010; 10: 200-205

38 Vuralkan E, Saka C, Akin I et al. Comparison of montelukast and mometasone furoate in the prevention of recurrent nasal polyps. Ther Adv Respir Dis 2012; 6: 5-10

39 Carta F, Blancal JP, Verillaud B et al. Surgical management of inverted papilloma: approaching a new standard for surgery. Head Neck 2012: epub ahead of print

40 Gurr A, Stark T, Noack V et al. Ein malignes Melanom der Kieferhöhle - ein Fallbericht. Laryngo-Rhino-Otologie 2009; 88: 35-38

41 Tack D, Widelec J, De Maertelaer V et al. Comparison between low-dose and standard-dose multidetector CT in patients with suspected chronic sinusitis. AJR 2003; 181: $939-944$

42 Briner HR, Simmen D, Jones N. Endoscopic sinus surgery: advantages of the bimanual technique. Am I Rhino 2005; 19: 269-273

43 Al Kadah B, Bumm K, Charalampaki P et al. Erste Erfahrungen in der endonasalen Chirurgie mit einem neuen 3DChipendoskop. Laryngo-Rhino-Otolologie 2012; 91 : $428-433$

44 Caversaccio $M$, Zheng $G$, Nolte LP. Computerassistierte Chirurgie der Nasennebenhöhlen und der vorderen Schädelbasis. HNO 2008; 56: 376-382

45 Simmen $D$, Jones $N$. Chirurgie der Nasennebenhöhlen und der vorderen Schädelbasis. 1. Auflage. Stuttgart: Thieme; 2005

46 Schnipper D, Spiegel JH. Management of intracranial complications of sinus surgery. Otolaryngol Clin North Am 2004; 37: $453-472$

47 Minovi A, Hummel T, Ural A et al. Predictors of the outcome of nasal surgery in terms of olfactory function. Eur Arch Otorhinolaryngol 2008; 265: 57-61

48 Jorissen M, Bachert C. Effect of corticosteroids on wound healing after endoscopic sinus surgery. Rhinology 2009; 47: $280-286$

49 Bassiouni A, Naidoo Y, Wormald PJ. When FESS fails: the inflammatory load hypothesis in refractory chronic rhinosinusitis. Laryngoscope 2012; 122: $460-466$

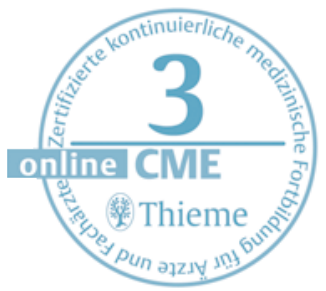




\section{CME-Fragen Chronische Rhinosinusitis ...}

1 Welche Aussage zur CRS ist nicht richtig?

A CRS ist ein häufiges Krankheitsbild mit hoher sozioökonomischer Bedeutung.

B Im Rahmen der Abklärung von chronischem Husten sollte an eine CRS gedacht werden.

C CRS ist ein häufiges Problem in den ersten drei Lebensjahren.

D Ein typisches Symptom einer CRS ist der sogenannte postnasale Drip.

E Patienten mit zystischer Fibrose leiden oft unter CRS und sollten in regelmäßiger HNO-ärztlicher Behandlung sein.

Welche Aussage zur Rolle von Staphylokokken bei CRS ist nicht richtig?

A Staphylokokkenenterotoxine fungieren als Superantigene und induzieren eine polyklonale IgG-Synthese.

B Staphylokokken zählen zu den häufigen Erregern, die bei CRS eine Rolle spielen.

C Die Anwendung von Staphylokokken-wirksamer Mupirocin-Nasensalbe hat bei CRS keine anhaltenden positiven Effekte gezeigt.

D Staphylokokken können Biofilme verursachen.

E Staphylokokken gelten als sogenannte disease modifiers.

Welche Aussage zur antibiotischen Therapie bei CRS ist nicht richtig?

A Der Einsatz von Antibiotika zur Behandlung einer Exazerbation der CRS (AE-CRS) ist kritisch zu prüfen.

B Die Chinolone Moxifloxacin, Levofloxacin und Ciprofloxacin können zur antibiotischen Behandlung der AE-CRS verwendet werden.

C Zur antibiotischen Langzeittherapie der CRS können Makrolide in niedriger Dosierung eingesetzt werden.

D Makroliden werden antiinflammatorische Effekte zugeschrieben.

E Die Standardtherapiedauer mit Moxifloxacin bei AE-CRS sollte 14 Tage betragen.

4 Welche Aussage zur Therapie der CRS ist richtig?

A Für den Einsatz von Xylometazolin als Dekongestivum in der Therapie der CRS gibt es eine hohe Evidenz.

B Neben ätherischen Ölen zur Inhalation sind „Schleimlöser“ - sogenannte Sekretolytika ein bedeutender Pfeiler der CRS-Therapie.

C Die adaptive Desaktivierung bei Patienten mit CRS und Aspirin ${ }^{\circledR}$-sensitivem Asthma bronchiale sollte nach Möglichkeit als ambulante Leistung durch den hausärztlichen Bereich vorgenommen werden.

D Die frühe antibiotische Therapie bei AE-CRS ist über geringere Fehlzeiten im Berufsleben zu rechtfertigen.

E Die Wirksamkeit von Montelukast in der Behandlung der CRS muss an großen Patientenkollektiven bestätigt werden.
5 Welche Aussage zur CRS ist richtig?

A Für das Jahr 2013 wurde die Publikation einer revidierten Fassung der deutschen Leitlinie zur Rhinosinusitis angekündigt.

B Für die chronische Rhinosinusitis mit Nasenpolypen (CRSwNP) konnte ein Zusammenhang zur Besiedelung mit Pilzen nachgewiesen werden.

C Laryngopharyngealer Reflux zählt zu den Faktoren, die eine CRS unterhalten können.

D Ein Zusammenhang zwischen invasiver Beatmung via Tracheostoma und CRS wurde jüngst belegt.

E Bereits beim Vorliegen von typischen Beschwerden über einen Zeitraum von 10 Wochen liegt definitionsgemäß eine CRS vor.

6 Welche Aussage zur CRS trifft nicht zu?

A Bei rezidivierenden AE-CRS sollte an ein Immunmangelsyndrom gedacht werden.

B CRS zählt zu den Klassifikationskriterien des ChurgStrauss-Syndroms.

C Ein Zusammenhang zwischen CRS und der Einnahme von Montelukast wurde mehrfach angenommen.

D Die Computertomografie der Nasennebenhöhlen gehört zu den Tools, die im Rahmen der Diagnostik einer CRS zum Einsatz kommen können.

E In der Diagnostik der CRS ist die Magnetresonanztomografie der Computertomografie überlegen.

7 Welcher Erreger zählt nicht zu den häufigen Auslösern einer AE-CRS?

A Moraxella catarrhalis

B Pseudomonas aeruginosa

C Staphylococcus aureus

D Staphylococcus epidermidis

E Legionalla pneumophila

8 Welche der folgenden Aussagen ist richtig?

A Die Nasennebenhöhlenchirurgie ist ein besonders komplikationsarmes Teilgebiet der HNO-Heilkunde.

B Die konservative Therapie der CRS ist sinnlos, da in aktuellen Studien die Überlegenheit der NNH-Chirurgie nachgewiesen wurde.

C Für streng einseitige Befunde, auch mit knöchernen Arrosionen, gilt die 'wait and see'-Strategie.

D An die operativen Maßnahmen schließt sich nach Entfernung der Tamponaden eine Phase der intensiven Nachpflege der Nase an, die aus Absaugen und regelmäßigen Inhalationen besteht.

E Die Operationen der Nasennebenhöhlen müssen heute grundsätzlich unter Zuhilfenahme eines Operationsmikroskops erfolgen. Starr endoskopische Techniken sind veraltet. 
9 Welche der folgenden Aussagen ist richtig?

A Wichtigste Landmarke für einen Nasennebenhöhleneingriff ist der mittlere Nasengang.

B Die Eröffnung der Kieferhöhle ist schwierig und sollte erst zum Schluss des Eingriffs erfolgen.

C Die ambulante Polypektomie ist vor allem bei jungen Patienten zu indizieren, da diese weniger schmerzempfindlich sind.

D Bei schwieriger Anatomie ist die Eröffnung der Kieferhöhle über den unteren Nasengang indiziert.

E Auch bei geringer Ausprägung der CRS sollten immer alle Sinus operiert werden, um eine spätere Revisionschirurgie zu vereinfachen und das Rezidivrisiko zu senken.
10 Welche der folgenden Aussagen zu möglichen Komplikationen der Nasennebenhöhlenchirurgie ist nicht richtig?

A Postoperative Blutungen gehören zu den häufigeren Komplikationen einer NNH-OP.

B Die Verletzung der Schädelbasis ist eine schwerwiegende Komplikation. Sie muss umgehend intraoperativ versorgt werden.

C Die Schonung des Riechepithels ist unwichtig, da Patienten mit einer chronischen Rhinosinusitis grundsätzlich unter einer therapieresistenten Anosmie leiden.

D Bei Eingriffen am Keilbein kann es zu Verletzungen des Nervus opticus oder der Arteria carotis interna kommen.

E Der Patient muss präoperativ auch über orbitale Hämatome aufgeklärt werden 